\title{
Computational capacity of the universe
}

\author{
Seth Lloyd \\ d'Arbeloff Laboratory for Information Systems and Technology \\ MIT Department of Mechanical Engineering \\ MIT 3-160, Cambridge, Mass. 02139 \\ slloyd@mit.edu
}

\begin{abstract}
Merely by existing, all physical systems register information. And by evolving dynamically in time, they transform and process that information. The laws of physics determine the amount of information that a physical system can register (number of bits) and the number of elementary logic operations that a system can perform (number of ops). The universe is a physical system. This paper quantifies the amount of information that the universe can register and the number of elementary operations that it can have performed over its history. The universe can have performed no more than $10^{120}$ ops on $10^{90}$ bits.

'Information is physical'1. This statement of Landauer has two complementary interpretations. First, information is registered and processed by physical systems. Second, all physical systems register and process information. The description of physical systems in terms of information and information processing is complementary to the conventional description of physical system in terms of the laws of physics. A recent paper by the author ${ }^{2}$ put bounds on the amount of information processing that can be performed by physical systems.
\end{abstract}

The first limit is on speed. The Margolus/Levitin theorem ${ }^{3}$ implies that the total number of elementary operations that a system can perform per second is limited by its energy: \#ops/sec $\leq 2 E / \pi \hbar$, where $E$ is the system's average energy above the ground state and $\hbar=1.0545 \times 10^{-34}$ joule-sec is Planck's reduced constant. As the presence of Planck's constant suggests, this speed limit is fundamentally quantum mechanical, ${ }^{2-4}$ and as shown in (2), is actually attained by quantum computers, ${ }^{5-27}$ including existing 
devices. ${ }^{15,16,21,23-27}$

The second limit is on memory space. The total number of bits available for a system to process is limited by its entropy: \# bits $\leq S / k_{B} \ln 2$, where $S$ is the system's thermodynamic entropy and $k_{B}=1.38 \times 10^{-23}$ joule $/ \mathrm{K}$ is Boltzmann's constant. ${ }^{2}$ This limit, too, is attained by existing quantum computers, which store one bit of information per nuclear spin or photon polarization state.

The speed at which information can be moved from place to place is limited by the speed of light, $c=2.98 \times 10^{8}$ meters/sec. This limit can be combined with the first two to limit the input/output rate of computational systems. ${ }^{2}$ The maximum rate at which information can be moved in and out of a system with size $R$ is $\approx c S / k_{B} R$ (attained by taking all the information $S / k_{B} \ln 2$ in the system and moving it outward at the speed of light). The ratio between the maximum rate of information processing (ops/sec) and the input/output rate (bits in/out per second) is $\approx k_{B} E R / \hbar c S$, a quantity shown by Bekenstein to be $\geq 1 / 2 \pi$, with equality attained for black holes. ${ }^{28-30}$

Here, these three limits are applied to calculate the information processing capacity of the universe. In particular, I calculate both the total number of bits available in the universe for computation, and the the number of elementary logical operations that can have been performed on those bits since the universe began. Perhaps not surprisingly, the total number of bits $\left(\approx 10^{90}\right.$ in matter, $\approx 10^{120}$ if gravitation is taken into account) and ops $\left(\approx 10^{120}\right)$ turn out to be simple polynomials in the fundamental constants of nature, Planck's constant $\hbar$, the speed of light $c$ and the gravitational constant $G=6.673 \times 10^{-11}$ meters $^{3} /$ kilogram sec ${ }^{2}$, together with the current age of the universe. These numbers of ops and bits can be interpreted in three distinct ways:

1. They give upper bounds to the amount of computation that can have been performed by all the matter in the universe since the universe began.

2. They give lower bounds to the number of ops and bits required to simulate the entire universe on a quantum computer.

3. If one chooses to regard the universe as performing a computation, these numbers give the numbers of ops and bits in that computation.

The first two interpretations are straightforward: even if all the matter in the universe were organized in a computer, that computer could not register more information or perform more elementary logical operations than calculated here. Similarly, a quantum computer 
that accurately simulates the time evolution of the universe requires at least this number of bits and must perform at least this number of operations. ${ }^{18-20}$ The third interpretation is potentially more controversial. ${ }^{31}$ It is well established that all degrees of freedom can be taken to register information and all physical dynamics transforms that information: every nuclear spin can be thought of as registering a bit, and when the spin flips from clockwise to counterclockwise, the bit flips. Similarly, it is known that fundamental interactions between elementary particles allow the performance of quantum logic operations. ${ }^{12-16}$ According to the third interpretation, every particle in the universe registers one or more bits, and every time those particles interact, they perform one or more elementary operations. These interpretations will be discussed in greater detail below.

Now let us calculate the computational capacity of the universe. For clarity of exposition, the calculation will not explicitly keep track of factors of $1 / 2, \pi$, etc., that only affect the final results by an order of magnitude or so (i.e., a factor of $2 \pi$ will be ignored; a factor of $(2 \pi)^{6}$ will not be). The expression $\approx$ will be used to indicate equality to within such factors. In other words, $X \approx Y$ is equivalent to $\log X=\log Y+O(1)$.

To calculate the number of bits available and the number of ops that can have been performed over the history of the universe requires a model of that history. The standard big-bang model will be used here. ${ }^{32}$ In this model the universe began $\approx 10^{10}$ years ago in what resembled a large explosion (the big bang). Since the big bang, the universe has expanded to its current size. The big bang model successfully explains a number of features of the current universe, such as its ongoing expansion and the existence of the microwave background. Discussions of the amount of computation that can have been performed in the pre-big bang universe are necessarily speculative: the well-established inflationary scenario will be used to investigate computation in this regime. ${ }^{33-34}$

For most of its history, the universe has been matter-dominated - most of the energy is in the form of matter. As will be seen below, most of the computation that can have taken place in the universe occurred during the matter-dominated phase. Accordingly, begin with the computational capacity of the matter-dominated universe, and then work back through the radiation-dominated and inflationary universe.

\section{Number of ops}

First, investigate the number of elementary logic operations that can have been performed. As noted above, the maximum number of operations per second that can be performed by a physical system is proportional to its energy. This result follows from the 
Margolus-Levitin theorem, which states that the minimum time required for a physical system to move from one state to an orthogonal state is given by $\Delta t=\pi \hbar / 2 E$, where $E$ is the average energy of the system above its ground state. ${ }^{3}$

The Margolus-Levitin theorem is a mathematical expression of the familiar fact that energy must be invested to change a system such as a quantum bit from one state to another. As an example, consider the process of flipping a bit in an NMR quantum computer. In NMR quantum computing, each nuclear spin in a molecule registers a quantum bit, with spin up identified with 0 and spin down identified with 1. To flip a quantum bit, apply a magnetic field. The spin precesses about the magnetic field: when the spin has precessed by an angle $\pi$, the state was spin up has become spin down and vice versa. In terms of bits, 0 has become 1 and 1 has become 0: flipping the spin flips the bit. It is straightforward to verify that the spin/bit flipping process takes place in time $\Delta t=\pi \hbar / 2 E=\pi / \omega_{L}$, where $E$ is the average energy of the spin's interaction with the magnetic field and $\omega_{L}$ is the spin's Larmor frequency. Two-qubit quantum logic operations obey the same bound, with $E$ the average energy of interaction between the qubits. Note that while energy must be invested in the spin-field interaction to flip the bit, it need not be dissipated.

The Margolus-Levitin bound also holds for performing many logic operations in parallel. If energy $E$ is divided up among $N$ quantum logic gates, each gate operates $N$ times more slowly than a single logic gate operating with energy $E$, but the maximum total number of operations per second remains the same.

Now apply these results to the universe as a whole. In the matter-dominated universe, the energy within a co-moving volume is approximately equal to the energy of the matter within that volume and remains approximately constant over time. (A co-moving volume is one that is at rest with respect to the microwave background, and that expands as the universe expands.) Since the energy remains constant, the number of ops per second that can be performed by the matter in a co-moving volume remains constant as well. The total volume of the universe within the particle horizon is $\approx c^{3} t^{3}$, where $t$ is the age of the universe. The particle horizon is the boundary between the part of the universe about which we could have obtained information over the course of the history of the universe and the part about which we could not. In fact, the horizon is currently somewhat further than ct away, due to the ongoing expansion of the universe, but in keeping with the approximation convention adopted above we will ignore this factor along with additional geometric factors in estimating the current volume of the universe. 
The total number of ops per second that can be performed in the matter-dominated universe is therefore $\approx \rho c^{2} \times c^{3} t^{3} / \hbar$, where $\rho$ is the density of matter and $\rho c^{2}$ is the energy density per unit volume. Since the number of ops per second in a co-moving volume is constant, and since the universe has been matter dominated for most of its history, we have

$$
\# \mathrm{ops} \approx \rho c^{5} t^{4} / \hbar
$$

Insertion of current estimates for the density of the universe $\rho \approx 10^{-27} \mathrm{~kg} / \mathrm{m}^{3}$ and the age of the universe $t \approx 10^{10}$ years, we see that the universe could have performed $\approx 10^{120}$ ops in the course of its history.

A more revealing form for the number of ops can be obtained by noting that our universe is close to its critical density. If the density of the universe is greater than the critical density, it will expand to a maximum size and then contract. If the density is less than or equal to the critical density, it will expand forever. The critical density can be estimated by a simple, non-relativistic argument ${ }^{32}$. At the critical density, the kinetic energy of a galaxy at distance $R$ is equal to its gravitational energy, so that the galaxy has just enough energy to move to infinite distance. Galaxies at distance $R$ are moving away from our galaxy at a rate $\approx H R$, where $H \approx 1 / t$ is the Hubble constant. ( $H$ is somewhat smaller than $1 / t$ and is not in fact a constant, since the expansion is slowing over time. But $H \approx 1 / t$ to within the approximation convention adopted here.) The kinetic energy of a galaxy of mass $m$ at distance $R$ is therefore equal to $m H^{2} R^{2} / 2$, and its potential energy is $4 \pi G m \rho R^{2} / 3$. Equating the galaxy's kinetic energy with its potential energy yields the critical density $\rho_{c}=3 H^{2} / 8 \pi G \approx 1 / G t^{2}$.

So for a matter-dominated universe at its critical density, the total number of ops that can have been performed within the horizon at time $t$ is

$$
\text { \#ops } \approx \rho_{c} c^{5} t^{4} / \hbar \approx t^{2} c^{5} / G \hbar=\left(t / t_{P}\right)^{2}
$$

Here $t_{P}=\sqrt{G \hbar / c^{5}}=5.391 \times 10^{-44}$ seconds is the Planck time - the time scale at which gravitational effects are of the same order as quantum effects.

In retrospect, it should come as no surprise that the number of elementary operations that can have been performed within the horizon over the lifetime of a universe at critical density should be some polynomial in $t / t_{P}$. The number of operations per second is a function of the critical density $\rho_{c}$ and the age of the universe $t$, together with the fundamental constants. The total number of operations is a dimensionless number, and so 
should be a function of $t / \tau$, where $\tau$ is a timescale that is a function of $\hbar, c$, and $G$. But the only such timescale is the Planck time. The reason that the number of ops performed within the horizon grows as $\left(t / t_{P}\right)^{2}$ rather than simply $t / t_{P}$ is that the total amount of matter within the horizon is growing with time: as the universe gets older, more and more galaxies come into view.

The previous paragraphs calculate the maximum number of operations that could be performed by the matter in the universe. What about the number of elementary operations that could be performed by the gravitational field? Care must be taken in estimating this number, as the Margolus-Levitin theorem that allows the calculation of the number of operations performed by a physical system is strictly quantum-mechanical in nature, and no complete quantum theory of gravity currently exists. With this caveat in mind, however, one can still provisionally apply the theorem, which as noted above states that the number of operations per second that can be performed by a physical system is bounded above by $\approx E / \hbar$, where $E$ is the system's average energy above the ground state energy. The gravitational energy in the universe is negative and equal in magnitude to the positive energy of the matter in the universe, so that the total energy of the universe is zero. Since the Margolus-Levitin theorem enjoins the calculation of the number of ops per second using the average energy above the lowest energy that appears in the system's quantum state, the number of elementary operations that can be performed using gravitational energy is exactly equal to the number that can be performed using the energy in matter. Including the number of operations that can have been performed by gravitational energy (however one might accomplish such a feat!) then changes the overall number of operations by a factor of two. Even including gravitational energy, the total number of operations that can have been performed in the universe is $\approx\left(t / t_{P}\right)^{2} \approx 10^{120}$.

It is instructive to compare the total number of operations that could have been performed using all the matter in the universe with the number of operations that have been performed by conventional computers. The actual number of elementary operations performed by all man-made computers is of course much less than this number. Because of Moore's law, about half of these elementary operations have been performed in the last two years. Let us estimate the total number of operations performed by man-made computers, erring on the high side. With $\approx 10^{9}$ computers operating at a clock rate of $\approx 10^{9}$ Hertz performing $\approx 10^{5}$ elementary logical operations per clock cycle over the course of $\approx 10^{8}$ seconds, all the man-made computers in the world have performed no more than $\approx 10^{31}$ 
ops over the last two years, and no more than approximately twice this amount in the history of computation.

What is the universe computing? In the current matter-dominated universe most of the known energy is locked up in the mass of baryons. If one chooses to regard the universe as performing a computation, most of the elementary operations in that computation consists of protons, neutrons (and their constituent quarks and gluons), electrons and photons moving from place to place and interacting with each other according to the basic laws of physics. In other words, to the extent that most of the universe is performing a computation, it is 'computing' its own dynamical evolution. Only a small fraction of the universe is performing conventional digital computations.

\section{Number of bits}

Information is registered by physical systems, and all physical systems can register information. ${ }^{1}$ The amount of information, measured in bits, that can be registered by any physical system is equal to the logarithm to the base 2 of the number of distinct quantum states available to the system given its overall energy, volume, electric charge, etc. ${ }^{2}$ In other words, $I=S / k_{B} \ln 2$, where $S$ is the maximum entropy of the system and $k_{B}=1.38 * 10^{-23}$ joule/ $K$ is Boltzmann's constant.

To calculate the number of bits that can be registered by the universe requires a calculation of its maximum entropy, a calculation familiar in cosmology. The maximum entropy in the matter-dominated universe would be obtained by converting all the matter into radiation. (Luckily for us, we are not at maximum entropy yet!) The energy per unit volume is $\rho c^{2}$. The conventional equation for black-body radiation can then be used to estimate the temperature $T$ that would be obtained if that matter were converted to radiation at temperature $T: \rho c^{2}=\left(\pi^{2} / 30 \hbar^{3} c^{3}\right)\left(k_{B} T\right)^{4} \sum_{\ell} n_{\ell}$. Here $\ell$ labels the species of effectively massless particles at temperature $T$ (i.e., $m_{\ell} c^{2}<<k_{B} T$ ), and $n_{\ell}$ counts the number of effective degrees of freedom per species: $n_{\ell}=$ (number of polarizations) * (number of particles/antiparticles) * 1 (for bosons) or $7 / 8$ (for fermions). Solving for the temperature for the maximum entropy state gives $k_{B} T=\left(30 \hbar^{3} c^{5} \rho / \pi^{2} \sum_{\ell} n_{\ell}\right)^{1 / 4}$. The maximum entropy per unit volume is $S / V=4 \rho c^{2} / 3 T$. The entropy within a volume $V$ is then $S=\left(4 k_{B} / 3\right)\left(\pi^{2} \sum_{\ell} n_{\ell} / 30\right)^{1 / 4}(\rho c / \hbar)^{3 / 4} V^{1 / 4}$. The entropy depends only weakly on the number of effectively massless particles.

Using the formula $I=S / k_{B} \ln 2$ and substituting $\approx c^{3} t^{3}$ for the volume of the universe 
gives the maximum number of bits available for computation:

$$
I \approx\left(\rho c^{5} t^{4} / \hbar\right)^{3 / 4}=(\# \mathrm{ops})^{3 / 4}
$$

The universe could currently register $\approx 10^{90}$ bits. To register this amount of information requires every degree of freedom of every particle in the universe.

The above calculation estimated only the amount of information that could be stored in matter and energy and did not take into account information that might be stored on gravitational degrees of freedom. The calculation of the amount of information that can be stored by a physical system is intrinsically quantum mechanical. Accordingly, just as in the calculation of the number of operations that could be performed by the gravitational field, an accurate account of the amount of information that can be registered gravitationally must await a full quantum theory of gravity. With this caveat in mind, note that the Bekenstein bound ${ }^{28-30}$ together with the holographic principle ${ }^{35-37}$ implies that the maximum amount of information that can be registered by any physical system, including gravitational ones, is equal to the area of the system divided by the the square of the Planck length, $\ell_{P}^{2}=\hbar G / c^{3}$. This limit is in fact attained by black holes and other objects with event horizons. Applying the Bekenstein bound and the holographic principle to the universe as a whole implies that the maximum number of bits that could be registered by the universe using matter, energy, and gravity is $\approx c^{2} t^{2} / \ell_{P}^{2}=t^{2} / t_{P}^{2}$. That is, the maximum number of bits using gravitational degrees of freedom as well as conventional matter and energy is equal to the maximum number of elementary operations that could be performed in the universe, $\approx 10^{120}$.

Not surprisingly, existing man-made computers register far fewer bits. Over-estimating the actual number of bits registered in 2001, as above for the number of ops, yields $\approx 10^{9}$ computers, each registering at $\approx 10^{12} \mathrm{bits}$, for a total of $\approx 10^{21} \mathrm{bits}$.

\section{Large Numbers}

Three quarters of a century ago, Eddington noted that two large numbers that characterize our universe happen to be approximately equal. ${ }^{38}$ In particular, the ratio between the electromagnetic force by which a proton attracts an electron and the gravitational force by which a proton attracts an electron is $\alpha=e^{2} / G m_{e} m_{p} \approx 10^{40}$. Similarly, the ratio between the size of the universe and the classical size of an electron is $\beta=c t /\left(e^{2} / m_{e} c^{2}\right) \approx 10^{40}$. The fact that these two numbers are approximately equal is currently regarded as a coincidence, although Dirac constructed a theory in which they are equal by necessity. Dirac's theory is not favored by observation. 
A third large number, the square root of the number of baryons in the universe, $\gamma=\sqrt{\rho c^{3} t^{3} / m_{p}}$ is also $\approx 10^{40}$. This is not a coincidence given the values of $\alpha$ and $\beta$ : $\alpha \beta \approx \gamma^{2}$ in a universe near its critical density $\rho_{c} \approx 1 / G t^{2}$.

The astute reader may have noted that the number of operations that can have been performed by the universe is approximately equal to the Eddington-Dirac large number cubed. In fact, as will now be shown, the number of ops is necessarily approximately equal to $\beta \gamma^{2} \approx \alpha \beta^{2} \approx 10^{120}$. This relation holds true whether or not $\alpha \approx \beta \approx \gamma$ is a coincidence. In particular,

$$
\beta \gamma^{2}=\left(\rho c^{5} t^{4} / \hbar\right)\left(\hbar c / e^{2}\right)\left(m_{e} / m_{p}\right)=\# \text { ops } *(137 / 1836)
$$

Similarly,

$$
\alpha \beta^{2}=\left(t / t^{P}\right)^{2}\left(\hbar c / e^{2}\right)\left(m_{e} / m_{p}\right)=\# \text { ops } *(137 / 1836)
$$

That is, the number of ops differs from the Eddington-Dirac large number cubed by a factor of the fine structure constant times the proton-electron mass ratio. Since the number of

ops is $\approx 10^{120}$, as shown above, and the fine-structure constant times the proton-electron mass ratio is $\approx 10$, the number of ops is a factor of ten larger than the Eddington-Dirac large number cubed.

In other words, whether or not the approximate equality embodied by the EddingtonDirac large number is a coincidence, the fact that the number of operations that can have been performed by the universe is related to this large number is not. These numerical relations are presented here for the sake of completeness. The author encourages the reader not to follow in Dirac's footsteps and take such numerology too seriously.

\section{Computation in the radiation-dominated universe}

The energy density of the universe became dominated by matter about 700, 000 years after the big bang. Before that time (with the exception of the first fraction of a second), the energy density of the universe was dominated by radiation, i.e., particles whose mass energy $m c^{2}$ is much less than their kinetic energy $k_{B} T$. In the radiation-dominated universe, the total energy in a co-moving volume is not constant. As the universe expands, the wavelength of the radiation is stretched by the scale factor $a$, which increases as $t^{1 / 2}$, so that the energy in an individual photon or particle of radiation is decreased by the same factor. Accordingly, if the energy in the radiation-dominated universe available for computation at time $t_{1}$ is $E$, the energy available at an earlier time $t_{0}$ is $E\left(t_{1} / t_{0}\right)^{1 / 2}$. Note that this result is independent of the total number of species of massless particles as long 
as no phase transition takes place in which a species of particle goes from being effectively massless to massive between $t_{0}$ and $t_{1}$.

In other words, as $t \rightarrow 0$, the total energy available for computation in the radiationdominated universe diverges. Rephrased in computational terms, the number of operations per second that can be performed in the radiation-dominated universe diverges as $t \rightarrow 0$. At first, this fact might seem to imply that the total number of operations performed in the radiation-dominated universe is formally infinite. However, as will now be seen, this is not the case.

The total number of operations that can have been performed within a co-moving volume is

$$
\text { \#ops }=(2 E / \pi \hbar) \int_{t_{0}}^{t_{1}}\left(t_{1} / t\right)^{1 / 2} d t=(4 E / \pi \hbar)\left(t_{1}-\sqrt{t_{1} t_{0}}\right) .
$$

But since $2\left(t_{1}-t_{0}\right) \geq 2\left(t_{1}-\sqrt{t_{1} t_{0}}\right) \geq t_{1}-t_{0}$, the total number of operations that can have been performed in the radiation-dominated universe over a time interval $t_{1}-t_{2}$ in a co-moving volume with energy $E$ at time $t_{1}$ is no more than twice the number that can have been performed in the matter-dominated universe in a co-moving volume with the same energy over the same amount of time. Accordingly, the formulae for the number of operations derived above for the matter-dominated universe also hold for the radiationdominated universe to within the accuracy of our approximation.

This result - that the amount of information processing in the radiation-dominated universe is finite even as $t \rightarrow 0$ - provides a partial answer to a question posed by Dyson, ${ }^{39}$ who addressed the problem of the amount of information processing possible during the 'big crunch.' The big crunch is the time inverse of the big bang, in which a closed universe contracts to a singularity. The results derived here for the number of operations and number of bits available for computation hold equally for the big bang and the big crunch. Equation (5) shows that the amount of computation that can be performed in the radiation-dominated universe is finite, whether during the big bang or the big crunch. To perform an infinite number of operations would require that the amount of energy in a co-moving volume goes to infinity at a rate $1 / t$ or faster as $t \rightarrow 0$. As will be seen below, quantum gravitational considerations make it unlikely that an infinite number of ops can be performed as $t \rightarrow 0$.

Now calculate the number of bits available in the radiation-dominated universe. The calculation is almost identical to that leading to equation (3), with the sole difference that here the dominant form of energy (the radiation) is already assumed to be in a maximum 
entropy state, unlike the matter in the matter-dominated universe. The number of bits available is

$$
I=S / k_{B} \ln 2=4 E / 3 \ln 2 k_{B} T=(4 / 3 \ln 2)(\# \text { ops })^{3 / 4} D^{1 / 4},
$$

where $D=\left(\pi^{2} / 30\right) \sum_{\ell} n_{\ell}$ (recall that $n_{\ell}$ is the number of effective degrees of freedom for the $\ell^{\prime}$ th species of effectively massless particle). As long as $k_{B} T$ is below the grand unification threshold of $2 \times 10^{16} \mathrm{GeV},\left(T \approx 10^{29} \mathrm{~K}\right)$, then $D^{1 / 4} \approx 1$, and the number of bits

available for computation is $\approx \# \mathrm{ops}^{3 / 4}$, just as in the matter-dominated universe. If one includes the number of bits that might be stored in gravitational degrees of freedom, then the total number of bits available is $\approx\left(t / t_{P}\right)^{2} \approx \#$ ops, just as in the matter-dominated universe.

The formulae for the number of elementary operations and number of bits available are essentially the same for the radiation-dominated universe and the matter-dominated universe. That is not to say that the radiation- and matter-dominated universes are computationally similar in all respects. The matter-dominated universe is far from thermal equilibrium: most of the energy is in the form of free energy, which is available for conversion to useful work via, e.g., nuclear fusion. Most of the matter is in the form of the quarks and gluons that provide the structural stability of baryons. In the radiation-dominated universe, by contrast, most of the energy is in the form of effectively massless particles at thermal equilibrium so that there is little free energy available and structural stability is hard to maintain. In other words, the matter-dominated universe is a much more friendly environment for conventional digital computation, not to mention for life as we know it.

\section{Computation in the inflationary universe}

The results derived above put limits on the amount of computation that can have taken place in the universe from the Big Bang on. The dynamics of the Big Bang is governed by the Standard Model of elementary particles, which is well-established to temperatures of at least $k_{B} T \approx 100 \mathrm{GeV}$, corresponding to a time of no more than $10^{-10}$ seconds after the universe began. How far back the Big Bang extends, and what happened before the Big Bang is not precisely known. In addition, although the Big Bang model accurately describes features of the observed universe such as its expansion, the abundances of light elements, and the cosmic microwave background, it fails to account for the universe's observed flatness, homogeneity, and isotropy (as well as other features such as the scarcity of magnetic monopoles). Put more colloquially, the Big Bang model does not account for the observed fact that the night sky looks qualitatively the same in all directions: in the 
standard Big Bang model, sufficiently distant pieces of the observed universe were never in causal connection and so have no particular reason to be similar.

This apparent difficulty is resolved by recently developed inflationary cosmologies. ${ }^{33-34}$ In these cosmologies, the scale factor for the universe grows at an exponential rate for some period of time, effectively 'stretching out' the universe from an initially small, causally connected volume to a large, causally unconnected volume. Since the observed universe now originates from a causally connected volume, its homogeneity and isotropy can be explained (as well as its flatness, the lack of magnetic monopoles, etc.). The details of the inflationary scenario depend on physics beyond the standard model, but its predictions are sufficiently compelling that it has been adopted by many cosmologists as a promising provisional theory of the very early universe.

The amount of computation that can have been performed during the inflationary universe is readily calculated. Inflation occurs when the scale factor $a$ of the universe is accelerating: $\ddot{a}>0$. The Hubble parameter $H=\dot{a} / a$ measures the rate of expansion of the universe, as above. For simplicity, concentrate on a universe at critical density. For such a universe, $H=\sqrt{8 \pi G \rho_{c} / 3 c^{2}}$, as above. In the very early universe, the energy density $\rho_{c}$ could be supplied by a scalar field (the 'inflaton') or by an effective cosmological constant. During inflation, the scale factor grows exponentially at a rate $H$. Such a universe locally appears to be a De Sitter space and possesses an horizon at distance $\approx c / H$ : an inertial observer sees all matter accelerating away at a rate proportional to its distance. The energy within the horizon is $\approx \rho_{c} c^{3} / H^{3}$ and the total number of elementary logical operations that can be performed per second within the horizon is $\approx \rho_{c} c^{3} / H^{4} \hbar \approx 1 / t_{P}^{2} H$. Similarly, the total number of ops that can be performed within the horizon in a time $t \approx 1 / H$ is $\approx 1 / t_{P}^{2} H^{2}$. In other words, the amount of computation that can be performed within the horizon in the Hubble time is $t^{2} / t_{P}^{2}$, as in the critical matter-dominated and radiationdominated universes. By the holographic principle, the maximum information that can be stored within the horizon is equal to the horizon area $\approx c^{2} / H^{2}$ divided by the Planck length squared. So the number of bits that can be registered within the horizon is no greater than $t^{2} / t_{P}^{2}$, as well.

As just shown, the formulae derived for number of bits and number of ops in a universe at critical density hold during inflation. The horizon scale is thought to go from the Planck scale at inflation to a scale between $10^{10 \pm 6}$ times larger at the end of inflation. Accordingly, the universe can have performed no more than $10^{20 \pm 12}$ ops on the same number of bits 
within the horizon during inflation (the large uncertainties reflect the current theoretical uncertainties in the inflationary scenario). Inflation is followed by a period of matter creation and reheating leading up to the start of the hot Big Bang. The formulae derived for the universe at critical density hold for this stage as well.

Even though the amounts of computation that can have been performed during inflation are relatively small, there is one highly significant information-based process that occurs during inflation. Because the scale factor for the universe increases by $\approx 50 e$-foldings $\left(e^{50}\right)$, the volume of the initially causally connected section of the universe increases by $\approx 150 e$-foldings. The resulting volume consists of causally unconnected sectors (separated by horizons as long as inflation persists). Although these sectors cannot participate in a joint computation (they cannot communicate with eachother), they have a much higher free energy and so a much higher maximum entropy than the initial volume. In other words, the exponential expansion can be regarded as a massive 'bit-creation' process. Indeed, Zizzi has suggested that inflation be regarded as adding new bits to a quantum computer, with the computation provided by the action of quantum gravity. ${ }^{35-36}$ Recent results suggest that quantum gravitational systems can be thought of in terms of quantum computation, ${ }^{2,35-37}$ a striking example of Wheeler's slogan, 'It from Bit.' ${ }^{40}$ Quantum gravity provides a maximum energy density given by the Planck scale and a maximum bit density supplied by the holographic principle. Accordingly, the amounts of computation that can be performed in a quantum gravitational context are finite. Indeed, the fundamentally Planck-scale character of the limits derived above suggests that they hold equally in quantum gravity. But whether or not these limits hold for the first instant of the universe's existence is a question, like many raised here, whose answer must await the construction of a consistent theory of quantum gravity.

\section{Discussion}

The above sections calculated how many elementary logical operations that can have been performed on how many bits during various phases of the history of the universe. As noted above, there are three distinct interpretations of the numbers calculated. The first interpretation simply states that the number of ops and number of bits given here are upper bounds on the amount of computation that can have been performed since the universe began. This interpretation should be uncontroversial: existing computers have clearly performed far fewer ops on far fewer bits. As Moore's law progresses and as computers use fewer degrees of freedom per bit and less energy per logic operation, the 
number of bits and ops available will increase. Existing quantum computers already use the minimum number of degrees of freedom per bit and the minimum energy per operation. The maximum amount of computing power that will eventually be available to mankind is a question of considerable technological and social interest. ${ }^{41}$ Of course, that maximum computing power is likely to remain far below the amounts calculated for the universe as a whole.

The second interpretation notes that the numbers calculated give a lower bound on the number of bits and the number of operations that must be performed by a quantum computer that performs a direct simulation of the universe. This interpretation should also be uncontroversial: quantum computers have been shown to be efficient simulators of any physical system that evolves according to local interactions. ${ }^{18-20} \mathrm{In}$ particular, a quantum computer can simulate the time evolution of any quantum system to an arbitrary degree of accuracy using the same number of degrees of freedom as the system, and a number of operations that is proportional to the time over which the system evolves. The universe is a physical system, and to simulate the time evolution of the universe, a quantum computer needs at least the same number of bits, and a number of operations at least as great as the total angle $\int E d t / \hbar$ evolved by the system's state in Hilbert space. Open questions include the efficiency and robustness of such simulations. For example, how much overhead in terms of ops and bits is required to simulate the standard model of elementary particles? This is a question that could be answered using current techniques. How hard is it to simulate quantum gravity on a quantum computer? This is a question whose answer must await the construction of a self-consistent theory of quantum gravity. But the fact that quantum computers can efficiently simulate any theory that is locally finite and that can be described by local strictly positive transformations ${ }^{18}$ is a strong indication that whatever the eventual theory of quantum gravity, it is efficiently simulatable on a quantum computer. A particularly striking demonstration of this last fact is Freedman's demonstration that quantum computers can efficiently simulate topological quantum field theories. ${ }^{42}$

The third interpretation — that the numbers of bits and ops calculated here represent the actual memory capacity and number of elementary quantum logic operations performed by the universe - is more controversial. That the universe registers an amount of information equal to the logarithm of its number of accessible states seems reasonable. But to say that $2 \int E d t / \pi \hbar$ is the number of elementary operations performed is equivalent to saying that the universe performes an 'op' every time some piece of it evolves by an 
average angle (or acquires an average phase) $\pi / 2$ in Hilbert space. In fact, for a quantum computer, this is a reasonable definition of an elementary quantum logic operations: to perform each quantum logic operation requires energy $E$ to be made available for a time $\pi \hbar / 2 E$. And whenever energy $E$ is available to some degrees of freedom for time $\pi \hbar / 2 E$, the state of those degrees of freedom evolves by an angle $\pi / 2$ in Hilbert space. In addition, almost any interaction between degrees of freedom, including fundamental interactions such as those in the standard model, supports quantum logic and quantum computation. ${ }^{12-16,43}$ Accordingly, it seems plausible to identify an elementary quantum logic operation with the local evolution of information-carrying degrees of freedom by an angle of $\pi / 2$. Whether or not this is a reasonable definition of an elementary operation for the universe as a whole is a question whose answer will have to await further results on the relationship between information processing and fundamental physics.

Is the universe a computer? The answer to this question depends both on the meaning of 'computer' and on the meaning of 'is.' On the one hand, the universe is certainly not a digital computer running Linux or Windows. (Or at any rate, not yet.) On the other hand, the universe certainly does represent and process information in a systematic fashion: as noted above, every spin can be taken to represent a bit, and every spin flip corresponds to a bit flip. Almost any interaction between degrees of freedom suffices to perform universal quantum logic on those degrees of freedom. ${ }^{12-13}$ Such universal interactions include the fundamental interactions of quantum electrodynamics and of topological quantum field theories. ${ }^{12-16,42-43}$ These results strongly suggest that the universe as a whole is capable of universal quantum computation. In such a 'universal' universal computer, every degree of freedom in the universe would register information, and the dynamics of those degrees of freedom would process that information: the amount of information and the amount of information processing possible can be calculated using the formulae derived above. Even if the universe is not a conventional computer, it can still compute. 


\section{References:}

1. Landauer, R., Nature, 335, 779-784 (1988).

2. Lloyd, S., Nature, (2000).

3. Margolus, N., Levitin, L.B., in PhysComp96, T. Toffoli, M. Biafore, J. Leao, eds. (NECSI, Boston) 1996; Physica D 120, 188-195 (1998).

4. Peres, A., Quantum Theory: Concepts and Methods, (Kluwer, Hingham) 1995.

5. Benioff, P., J. Stat. Phys. 22, 563 (1980).

6. Feynman, R.P., Int. J. Theor. Phys. 21, 467 (1982).

7. Deutsch, D., Proc. Roy. Soc. Lond. A 400, 97 (1985).

8. Lloyd, S., Science, 261, pp. 1569-1571 (1993).

9. Shor, P., in Proceedings of the 35th Annual Symposium on Foundations of Computer Science, S. Goldwasser, Ed., IEEE Computer Society, Los Alamitos, CA, 1994, pp. 124-134.

10. Lloyd, S., Sci. Am. 273, 140 (1995).

11. Divincenzo, D., Science 270, 255 (1995).

12. Lloyd, S., Phys. Rev. Lett., 75, 346-349 (1995).

13. Deutsch, D., Barenco, A., Ekert, A., Proc. Roy. Soc. A, 449, 669-677 (1995).

14. Cirac, J.I., Zoller, P. Phys. Rev. Lett. 74, 4091-4094 (1995).

15. Turchette, Q.A., Hood, C.J., Lange, W., Mabuchi, H., Kimble, H.J., Phys. Rev. Lett., 75, 4710-4713 (1995).

16. Monroe, C., Meekhof, D.M., King, B.E., Itano, W.M., Wineland, D.J., Phys. Rev. Lett., 75, 4714-4717 (1995).

17. Grover, L.K., in Proceedings of the 28th Annual ACM Symposium on the Theory of Computing, ACM Press, New York, 1996, pp. 212-218.

18. Lloyd, S., Science, 273, 1073-1078 (1996).

19. Abrams, D., Lloyd, S., Phys. Rev. Lett. 81, 3992-3995, (1998).

20. Zalka, C., Proc. R. Soc. Lond A. Mat. 454, 313-322 (1998).

21. Cory, D.G., Fahmy, A.F., Havel, T.F., in PhysComp96, Proceedings of the Fourth Workshop on Physics and Computation, T. Toffoli, M. Biafore, J. Leão, eds., (New England Complex Systems Institute, Boston) 1996.

22. Gershenfeld, N.A., Chuang, I.L. Science 275, pp. 350-356 (1997).

23. Chuang, I.L., Vandersypen, L.M.K., Zhou, X., Leung, D.W., Lloyd, S., Nature 393, 143-146 (1998) May, 1998. 
24. Jones, J.A., Mosca, M., Hansen, R.H., Nature 393, 344-346 (1998).

25. Chuang, I.L., Gershenfeld, N., Kubinec, M., Phys. Rev. Lett. 80, 3408-3411 (1998).

26. Nakamura, Y., Pashkin, Yu.A., Tsai, J.S., Nature 398, 305 (1999)

27. Mooij, J.E., Orlando, T.P., Levitov, L., Tian, L., van der Wal, C.H., Lloyd, S., Science 285, 1036-1039 (1999).

28. Bekenstein, J.D., Phys. Rev. D. 23, 287 (1981).

29. Bekenstein, J.D., Phys. Rev. Letters 46, 623 (1981).

30. Bekenstein, J.D., Phys. Rev. D. 30, 1669-1679 (1984).

31. Pagels, H., The Cosmic Code: quantum physics as the language of nature, (Simon and Schuster, New York) 1982.

32. Peebles, P.J.E., Principles of Physical Cosmology, Princeton University Press, Princeton (1993).

33. Linde, A., Particle Physics and Inflationary Cosmology, Harwood Academic, New York (1990).

34. Liddle, A.R. and Lyth, D.H., Cosmological Inflation and Large-Scale Structure, Cambridge University Press, Cambridge (2000).

35. Zizzi, P., Entropy 2, 36-39 (2000).

36. Zizzi, P., 'The Early Universe as a Quantum Growing Network,' in Proceedings of IQSA Fifth Conference, March 31-April 5, 2001, Cesena-Cesenatico, Italy; Archive gr-qc/0103002.

37. Ng, J., Phys. Rev. Lett. 86, 2946-2947 (2001).

38. Eddington, A.S., The Mathematical Theory of Relativity, Cambridge University Press, Cambridge (1924).

39. Dyson, F., Rev. Mod. Phys. 51, 447-460 (1979).

40. Wheeler, J.A., in Complexity, Entropy, and the Physics of Information, W.H. Zurek, ed., Santa Fe Institute Studies in the Sciences of Complexity volume VIII, Addison Wesley, Redwood City (1988).

41. Fredkin, E., 'Ultimate Limits to Computation,' MIT Laboratory for Computer Science Seminar (2000).

42. Freedman, M.H., Kitaev, A., Wang, Z., 'Simulation of topological quantum field theories by quantum computers,' quant-ph/0001071.

43. Freedman, M.H., Larsen, M., Wang, Z., 'A modular functor which is universal for quantum computation,' quant-ph/0001108. 